\title{
0 \\ COVID-19 and complicated bacterial pneumonia in children
}

\section{To the Editor:}

We read with great interest the recent publication by STEINForT et al. [1] outlining the profound reduction in influenza infections in Australia as a result of social distancing during the COVID-19 pandemic. Similar dramatic effects of social distancing on paediatric hospital activity have been reported, with reduced hospital admissions, presentations for respiratory conditions and detection of other viruses [2-6]. However, the impact of the pandemic on bacterial respiratory infections has not been well characterised. The Australian state of Victoria (population 6.7 million) has experienced a prolonged period of social restrictions ("lockdown") since March 2020. This period spanned the southern hemisphere autumn to spring, the usual peak period for bacterial respiratory infections.

Here, we assessed admissions to The Royal Children's Hospital Melbourne (RCH) for thoracic empyema ("empyema"), a complication of bacterial pneumonia that requires inpatient management. For added context, admissions for bronchiolitis, a common and well-defined viral respiratory infection, and appendicitis, a common hospital-managed condition unlikely to be impacted by the lockdown, were assessed. RCH is the largest tertiary paediatric hospital in Victoria, with $>89000$ emergency department presentations and 52000 inpatient admissions annually.

Admissions from March to August 2020 were compared with the same months in the preceding 3 years. Patients with empyema aged 0-18 years were identified by International Classification of Disease (10th revision) discharge diagnosis. Every case was validated by manual file review using the electronic medical record and incorrectly coded cases were excluded (26 out of 113, 23.0\%). Discharge coding for bronchiolitis (0-12 months) and appendicitis (0-18 years) was validated for a subgroup of patients and found to be $>95 \%$ accurate. For each condition, Poisson regression was used to compare the number of admissions across the study years. The only covariate in the models was year of admission, which was treated as a categorical variable, with 2020 as the reference category. Analyses were performed using Stata version 16.1 (StataCorp, College Station, TX, USA). The RCH Human Research Ethics Committee approved the study (HREC/65077/RCHM-2020).

During lockdown, we observed a significant reduction in the cases of empyema (figure 1). A comparable reduction was seen for bronchiolitis but not for appendicitis, suggesting that our findings were unlikely to be due to changes in hospital presentation. These findings match the large reductions in influenza, respiratory syncytial virus, bronchiolitis and viral pneumonia cases reported by others during pandemic-related lockdown $[1,6,7]$. Elsewhere, a decline in streptococcal pharyngitis, acute otitis media and infectious mononucleosis has been noted [4]. Together, these data suggest a significant reduction in viral and bacterial respiratory tract infections. At the time of writing, the northern hemisphere is now approaching its usual peak season for respiratory infection. Further studies may determine the extent to which the reduction in empyema that we observed may be attributed to decreased bacterial transmission or a decisive role for viral infection in the pathogenesis of complicated bacterial pneumonia.

@ERSpublications

Social distancing measures instituted due to \#SARSCoV2 have dramatically reduced paediatric thoracic empyema cases in Australia https://bit.ly/3akG98M

Cite this article as: Kaddour M, Simeonovic M, Osowicki J, et al. COVID-19 and complicated bacterial pneumonia in children. ERJ Open Res 2021; 7: 00884-2020 [https://doi.org/10.1183/ 23120541.00884-2020].

Copyright $\odot$ The authors 2021. This version is distributed under the terms of the Creative Commons Attribution NonCommercial Licence 4.0. For commercial reproduction rights and permissions contact permissions@ersnet.org 
a)

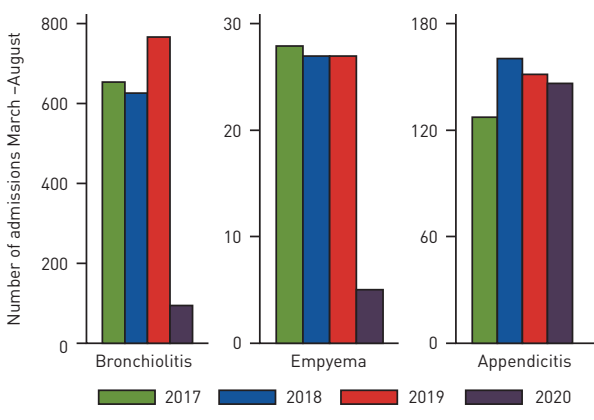

b)

\begin{tabular}{lcccc}
\hline Condition & Year & Number & $\begin{array}{c}\text { Incidence rate ratio } \\
(95 \% \text { Cl) }\end{array}$ & p-value \\
\hline Bronchiolitis & 2017 & 656 & $6.76(5.46-8.37)$ & $<0.001$ \\
& 2018 & 630 & $6.49(5.24-8.04)$ & \\
& 2019 & 768 & $7.92(6.41-9.78)$ & \\
& 2020 & 97 & Ref. & \\
Empyema & 2017 & 28 & $5.60(2.16-14.50)$ & 0.004 \\
& 2018 & 27 & $5.40(2.08-14.02)$ & \\
& 2019 & 27 & $5.40(2.08-14.02)$ & \\
& 2020 & 5 & Ref. & \\
Appendicitis & 2017 & 128 & $0.87(0.69-1.10)$ & 0.288 \\
& 2018 & 160 & $1.09(0.87-1.36)$ & \\
& 2019 & 152 & $1.03(0.82-1.30)$ & \\
& 2020 & 147 & Ref. & \\
\hline
\end{tabular}

FIGURE 1 a) Bar chart of bronchiolitis, empyema and appendicitis admissions occurring between March and August, 2017-2020. b) Results from Poisson regression models comparing the number of bronchiolitis, empyema and appendicitis admissions between March and August, 2017-2020.

Mohamad Kaddour ${ }^{1}$, Marcelle Simeonovic ${ }^{2}$, Joshua Osowicki ${ }^{3,4,5}$, Sarah McNab $^{2,5,6}$, Catherine Satzke ${ }^{5,7,8}$, Colin Robertson ${ }^{1,5,9}$, Cattram Nguyen ${ }^{5,10}$, Sebastian King ${ }^{5,11,12}$ and Shivanthan Shanthikumar $\oplus^{1,5,9}$

${ }^{1}$ Dept of Respiratory and Sleep Medicine, Royal Children's Hospital, Parkville, VIC, Australia. ${ }^{2}$ General Medicine, Royal Children's Hospital, Parkville, VIC, Australia. ${ }^{3}$ Infectious Diseases Unit, Dept of General Medicine, The Royal Children's Hospital, Parkville, VIC, Australia. ${ }^{4}$ Tropical Diseases, Murdoch Children's Research Institute, Parkville, VIC, Australia. ${ }^{5}$ Dept of Paediatrics, University of Melbourne, Parkville, VIC, Australia. ${ }^{6}$ Clinical Paediatrics, Murdoch Children's Research Institute, Parkville, VIC, Australia. ${ }^{7}$ Translational Microbiology, Murdoch Children's Research Institute, Parkville, VIC, Australia. ${ }^{8}$ Dept of Microbiology and Immunology, The University of Melbourne at the Peter Doherty Institute for Infection and Immunity, Parkville, VIC, Australia. ${ }^{9}$ Respiratory, Murdoch Children's Research Institute, Parkville, VIC, Australia. ${ }^{10}$ Infection and Immunity Theme, Murdoch Children's Research Institute, Parkville, VIC, Australia. ${ }^{11}$ Dept of Paediatric Surgery, Royal Children's Hospital, Melbourne, VIC, Australia. ${ }^{12}$ Surgical Research, Murdoch Children's Research Institute, Parkville, VIC, Australia.

Correspondence: Shivanthan Shanthikumar, Respiratory and Sleep Medicine, Royal Children's Hospital, 50 Flemington Road, Parkville, VIC, 3052, Australia. E-mail: shivanthan.shanthikumar@rch.org.au

Received: 26 Nov 2020 | Accepted: 22 Jan 2021

Conflict of interest: M. Kaddour has nothing to disclose. M. Simeonovic has nothing to disclose. J. Osowicki has nothing to disclose. S. McNab has nothing to disclose. C. Satzke reports being an investigator on a grant unrelated to this project from Pfizer. C. Robertson has nothing to disclose. C. Nguyen reports being an investigator on a grant unrelated to this project from Pfizer. S. King has nothing to disclose. S. Shanthikumar has nothing to disclose.

\section{References}

1 Steinfort DP, Cowie B, Johnson DF. Impact of general social distancing measures on incidence of influenza in Australia. ERJ Open Res 2020; 6: 00507-2020.

2 Vásquez-Hoyos P, Diaz-Rubio F, Monteverde-Fernandez N, et al. Reduced PICU respiratory admissions during COVID-19. Arch Dis Child 2020; in press [https://doi.org/10.1136/archdischild-2020-320469].

3 Scaramuzza A, Tagliaferri F, Bonetti L, et al. Changing admission patterns in paediatric emergency departments during the COVID-19 pandemic. Arch Dis Child 2020; 105: 704-706.

4 McBride JA, Eickhoff J, Wald ER. Impact of COVID-19 quarantine and school cancelation on other common infectious diseases. Pediatr Infect Dis J 2020; 39: e449-e452.

5 Kenyon CC, Hill DA, Henrickson SE, et al. Initial effects of the COVID-19 pandemic on pediatric asthma emergency department utilization. J Allergy Clin Immunol Pract 2020; 8: 2774-2776.e2771.

6 Wilder JL, Parsons CR, Growdon AS, et al. Pediatric hospitalizations during the COVID-19 pandemic. Pediatrics 2020; 146: e2020005983.

7 Britton PN, Hu N, Saravanos G, et al. COVID-19 public health measures and respiratory syncytial virus. Lancet Child Adolesc Health 2020; 4: e42-e43. 